\title{
IMPROVEMENT OF SC WIGGLER PERFORMANCE ${ }^{1}$ A. Mikhailichenko \\ Cornell University, LEPP, Ithaca NY 14853
}

\begin{abstract}
Described is a methodology of improvement for good field region in a SC 2.1 T CESR's wiggler as example. The method includes a tapering, shimming and pole face winding with active field distribution correction. This technology can be applied to any wiggler, however.
\end{abstract}

\section{INTRODUCTION}

Development of SC wigglers at Cornell LEPP was initiated by proposal considered to modernize the CESR for the low energy operation [1]. About 16 meters total of $\sim 2.1 \mathrm{~T}$ wigglers will bring CESR-c to $10^{32}$ level in luminosity. With installation and testing the first wiggler in CESR, the job started more than 2 years ago $[2,3]$ came to its end. In first run luminosity $L \geq 10^{31}$ achieved at $\Psi$, gives assurance, that program can be accomplished.

Meanwhile the concepts, procedures, technology and solutions developed for the SC wiggler during these years can be applied to any wiggler design.

Despite one purpose of this installation -damping time reduction -is similar to what required for future linear collider, the other one is different. Here the goal is to increase emittance in contrast to the goal clamed for the damping-ring in LC: as low emittance as possible. In both cases IBS will require large dynamic aperture however.

Wiggler is very tricky device, so its characteristics were recognized only recently. First systematic publication on nonlinear effects in a wiggler is probably [4], however it was not recognized as extremely important issue ${ }^{2}$. On the basis of [4] cautious estimations on influence of wiggler nonlinearities to damping ring performance were made in [5].

It was shown in [3], that physical nature of appearance of octupole (and higher) type dependence of kick versus vertical displacement is in wiggling with angle in fringe sextupole (and higher) field, as the fringe longitudinal field of any multipole has a structure of next order multipole. The sextupole generated intrinsically by the field variation in longitudinal direction (along the wiggler). When the pole is wide, the sextupole has strict value defined by second derivative of the wiggler field along the longitudinal coordinate $s$. For a pole with finite width the real sextupole adds (or subtracts) to the intrinsic one.

All this yields a requirement for a wide (and high) good field region. Fields in a wiggler need to be calculated with $3 \mathrm{D}$ code. That was recognized from the very beginning and first 3D field calculations with 3D code MERMAID ${ }^{3}$ were carried, allowing the field map usage for tracking through these fields. With the same code all optimizations for the coils sizes (3 types) and shapes were done, including a lot of more general work on shapes of poles, odd or even pole number, tapering and so on, $[2,3]$. And, of cause, the concept of the yoke shape itself was tested with this code.

\footnotetext{
${ }^{1}$ Electronic version is available at http://www.Ins.cornell.edu/public/CBN/2003/CBN03-3/CBN03_3.pdf

2 One can see this from the post talk questions after presentation [4] was made.

${ }^{3}$ MERMAID -MEsh oriented Routine for MAgnet Interactive Design, SIM Limited, Novosibirsk, P.O. Box 402, Russia.
} 
It was found that although the iron is saturated, it is possible to use iron profile for adjustment the flatness of the pole. Naturally this is possible in relatively narrow diapason of field variation. Fortunately in future linear collider the wigglers are planning to work at fixed field level. For CESR-c it was asked to have the diapason 1.7-2.1 Tesla, what made the task extremely difficult.

Nevertheless, the methods found might be interesting to others and now everyone can apply them to his own wiggler design.

\section{OPTIMAL DIMENSIONS}

Wiggler for CESR operation at $2 \mathrm{GeV}$ was described in [2,3]. As it was mentioned all calculations were carried with 3D code MERMAID.

Period of the wiggler is mostly important parameter. One can see this from the field representations [3]. In the case, when the pole width is much bigger, that its period, the field can be described by single generating function $B(s)$ only which stands for the vertical dipole field as

$$
\begin{gathered}
B_{y}(y, s)=B(s)-\frac{y^{2}}{2 !} B^{\prime \prime}(s)+\frac{y^{4}}{4 !} B^{(I V)}(s)-\ldots \\
B_{s}(y, s)=y \cdot B^{\prime}(s)-\frac{y^{3}}{3 !} B^{\prime \prime \prime}(s)+\frac{y^{5}}{5 !} B^{(V)}(s)-\ldots,
\end{gathered}
$$

where $y$ stands for vertical and $s$-for longitudinal coordinate, respectively. We would like to underline, that these are rectangular coordinates, associated with wiggler hardware, not curvilinear beam coordinates. Vertical field $B_{y}$ defines local amplitude of horizontal wiggling angle, $\alpha(s)=\int_{0}^{S} B_{y}(\sigma) d \sigma /(H R) / \operatorname{Cos} \alpha(\sigma) \cong \int B_{y}(\sigma) d \sigma /(H R)$, meanwhile $B_{s}$ defines forces acting to the particle in vertical direction $\propto \alpha \cdot B_{s}(s)$. One can see from (1), that gradient in $y$ direction associated with $G(s) \cong \alpha(s) B^{\prime}(s)$, octupole -with $O(s) \cong \frac{1}{6} \alpha(s) B^{\prime \prime \prime}(s)$. For example if dependence in $s$ direction is a periodic one, $B(s)=B_{0} \operatorname{Sin}\left(s / \lambda_{w}\right)$, then gradient in $y$ direction is

$$
\alpha(s) \cdot G(s) \equiv \alpha \frac{\partial B_{s}}{\partial y}=\alpha B^{\prime}(s)=\frac{\alpha \cdot B_{0}}{\lambda_{w}} \operatorname{Cos} \frac{s}{\lambda_{w}}, \quad k(s) \cong \alpha(s) G(s) /(H R),
$$

and the local octupole term goes to

$$
\alpha(s) \cdot O(s) \equiv \frac{\alpha}{3 !} \frac{\partial^{3} B_{y}}{\partial y^{3}}=-\frac{\alpha}{6} \frac{B_{0}}{\lambda_{w}^{3}} \operatorname{Cos} \frac{s}{\lambda_{w}} .
$$

Where $\alpha(s) \cong-\frac{B_{0} \lambda_{w}}{(H R)} \operatorname{Cos} \frac{s}{\lambda_{w}}$, and we neglected dependence of vertical field on vertical position. Integral over one period goes to be for octupole

$$
\int_{-\pi \lambda_{w}}^{\pi \lambda_{w}} \alpha(s) \cdot \boldsymbol{O}(s) d s \cong-\frac{1}{6(H R)} \frac{B_{0}^{2}}{\lambda_{w}^{2}} \int_{-\pi \lambda_{w}}^{\pi \lambda_{w}} \operatorname{Cos}^{2} \frac{s}{\lambda_{w}} d s=\frac{\pi}{6(H R)} \frac{B_{0}^{2}}{\lambda_{w}} .
$$

For the whole wiggler having length $L_{w}$, the number of acting gaps goes to be $N=\left(L / 2 \pi \lambda_{w}-1\right)$ and formula (4) gives full value of octupole integral over the wiggler as 


$$
\int_{0}^{L_{w}} \alpha(s) \cdot O(s) d s=\frac{\pi}{6(H R)} \frac{B_{0}^{2}}{\lambda_{w}}\left(\frac{L_{w}}{2 \pi \lambda_{w}}-1\right) \cong \frac{L_{w}}{12(H R)} \frac{B_{0}^{2}}{\lambda_{w}^{2}} .
$$

One can see from (1), that bending field is changing for sinusoidal longitudinal dependence as

$$
B_{y}(y, s) \cong B_{0} \cdot \operatorname{Sin}\left(\frac{s}{\lambda_{w}}\right) \cdot\left(1+\frac{y^{2}}{2 \lambda_{w}^{2}}+\frac{y^{5}}{5 ! \lambda_{w}^{5}} \ldots\right) .
$$

Formulas for wide poles (1) are valid for any longitudinal dependence, however. This knowledge might be important for proper modeling of entrance in the wiggler.

One can see, that the ratio of nonlinear field acting to the particle to the linear one at given amplitude $y_{0}$ as

$$
\frac{\alpha \cdot O(s) y_{0}^{3}}{\alpha \cdot G(s) y_{0}} \cong \frac{1}{6} \cdot \frac{y_{0}^{2}}{\hbar_{w}^{2}},
$$

indicates strong dependence on wiggler period. For the whole wiggler with fixed length, the net nonlinear effect defined by the number of poles and the field strength although the ratio (7) is not a function of magnetic field. All this initiating search of parameters within period values 30-40 $\mathrm{cm}$ region. Let's take for estimations $y_{0}=2 \mathrm{~cm}, \lambda_{w}=40 \mathrm{~cm}, \lambda_{w} \cong 6.37 \mathrm{~cm}$, so the ratio (7) goes to be $\sim 0.0164$ or $1.6 \%$, what is mentionable. Remember, quadrupole lenses typically designed and fabricated with this ratio at least ten times better.

Wiggler acts in one direction mostly, providing vertical focusing. As the particle entered with vertical coordinate, it goes closer to the medial plane at the out of the wiggler, where the field becomes weaker, (6). That is why it is difficult to keep orbit closed, as the wiggling amplitude is also changes with vertical position.

In case the poles are with finite width, additional functions required for proper description of the field. These are sextupole function, which manages quadratic terms in transverse dependence, decapole for terms with $x^{4}$ and so on. In practice these three functions describe all variety of situations in mostly cases. All these functions act in additional to intrinsic sextupole, decapole, ... associated with odd derivatives $S(s)=\frac{1}{8} B^{\prime \prime}(s), D(s)=\frac{1}{16} S^{\prime \prime}(s)-\frac{1}{192} B^{(I V)}(s), \ldots$ [3].

Instead (1) one can obtain the following presentation of fields in rectangular coordinates [3]

$$
\begin{aligned}
& B_{x}(x, y, s)=-\frac{x y}{4} B^{\prime \prime}(s)+2 S(s) x y+\ldots \\
& B_{y}(x, y, s)=B(s)+S(s)\left(x^{2}-y^{2}\right)-\frac{x^{2}+3 y^{2}}{8} B^{\prime \prime}(s)+\ldots \\
& B_{s}(y, s)=y \cdot B^{\prime}(s)-\frac{x^{2} y+y^{3}}{8} B^{\prime \prime \prime}(s)+\frac{3 x^{2}-y^{3}}{3} S^{\prime}(s)-\ldots .
\end{aligned}
$$

Sextupole in (8) includes intrinsic one, i.e. it is full sextupole. In curvilinear coordinates having bending radius $\rho=(H R) / B_{y}(0,0, s)$ the terms with gradient $G(\mathrm{~s})$ need to be included in these expansions ${ }^{4}$.

Typical mistake here often appeared in some publications is that the roll of the field at the top of the pole is treated as pure sextupole.

\footnotetext{
${ }^{4}$ J.F.Streib, HEPL 104, Stanford University (1960).
} 
With MERMAID 3D not only dimensions and shapes of coils were optimized, but the structure of wiggler in more general sense. Such as closed or not closed yoke, poles configuration etc. Some of them are not trivial in nature, such as shape and cross section of coils, for example.
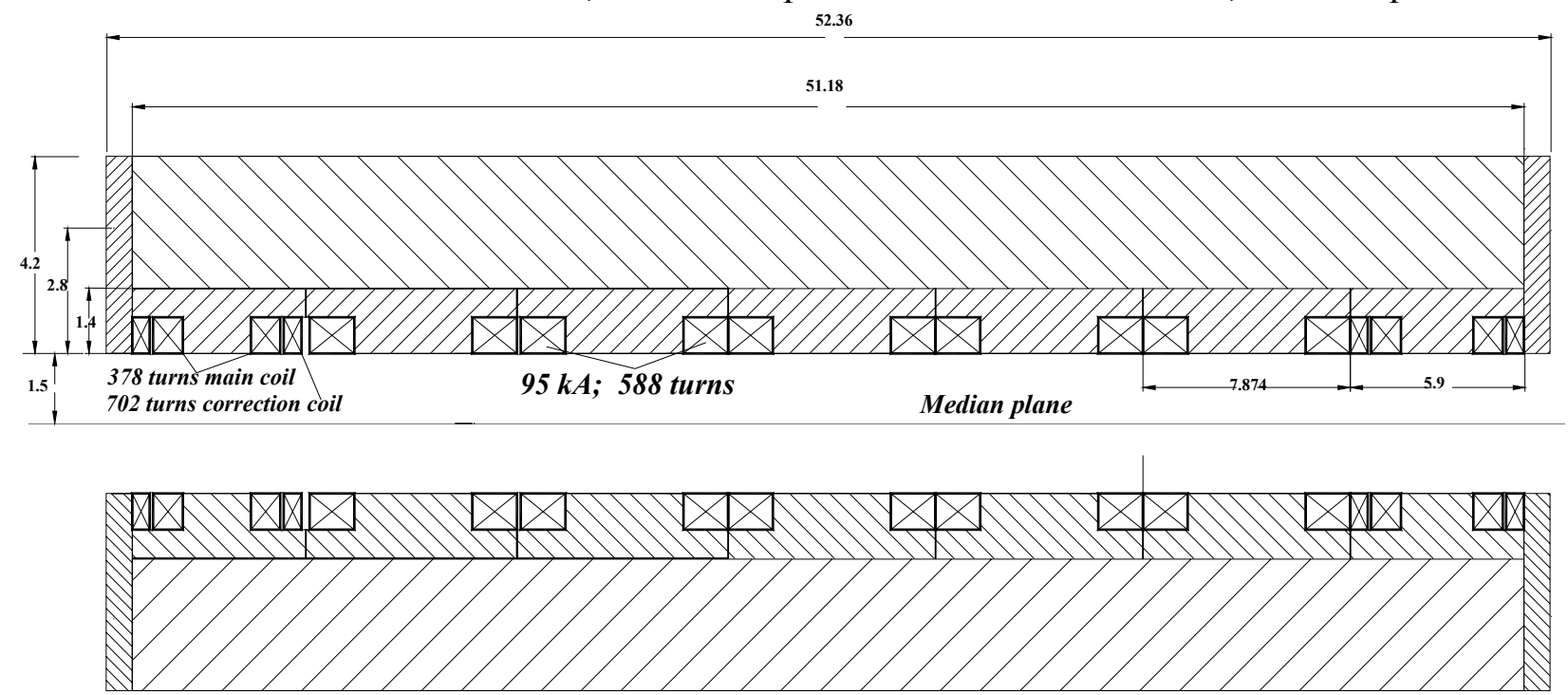

FIGURE 1: Dimensions of 40- $\mathrm{cm}$ period 7-pole wiggler \#1. Wiggler \#2 and all others have 660 turns of 0.8-mm OXFORD wire in main coil. A-turns remain the same however.

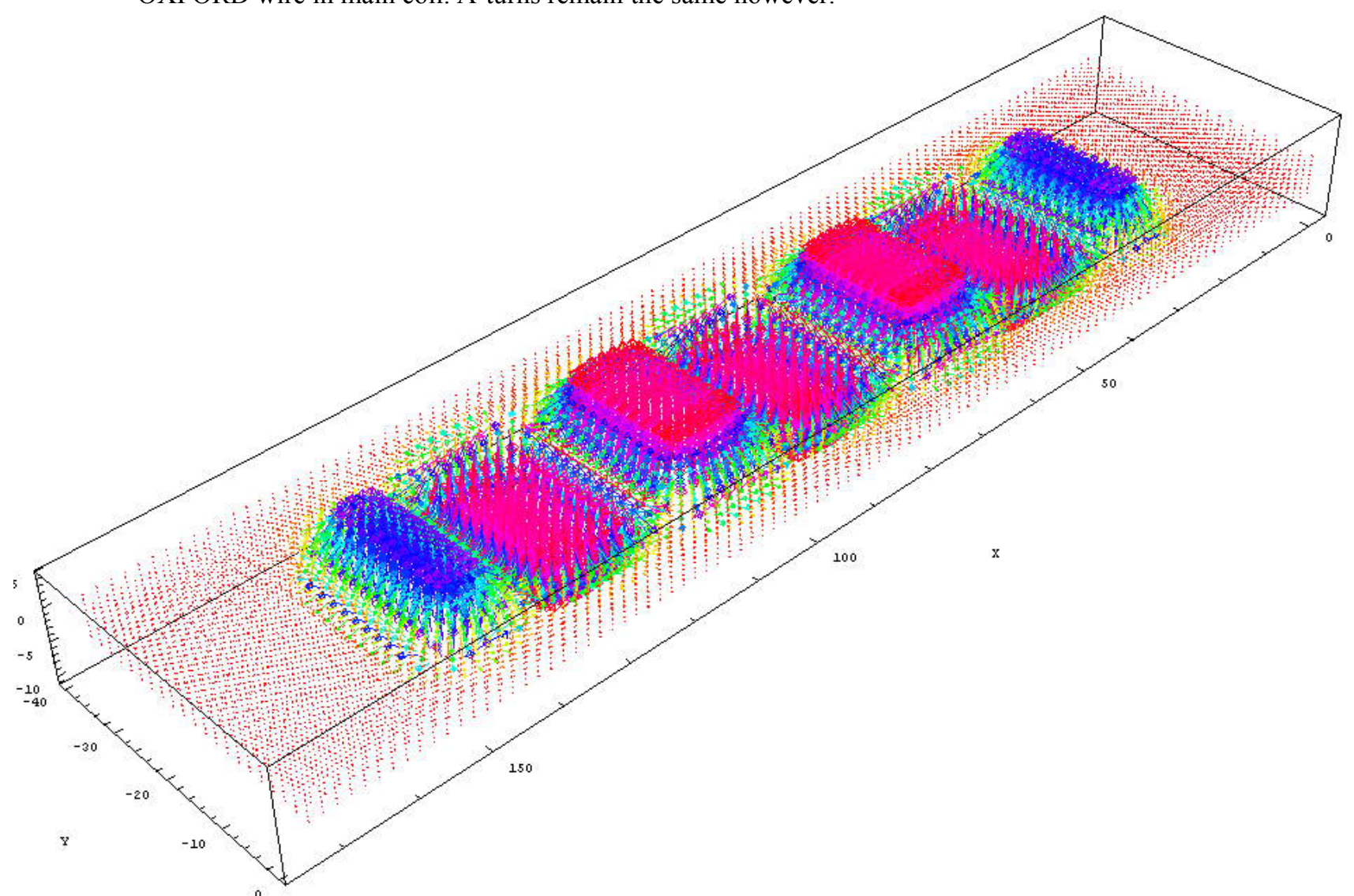

FIGURE 2: (Color) Vectorial representation of fields in 7-pole wiggler. 


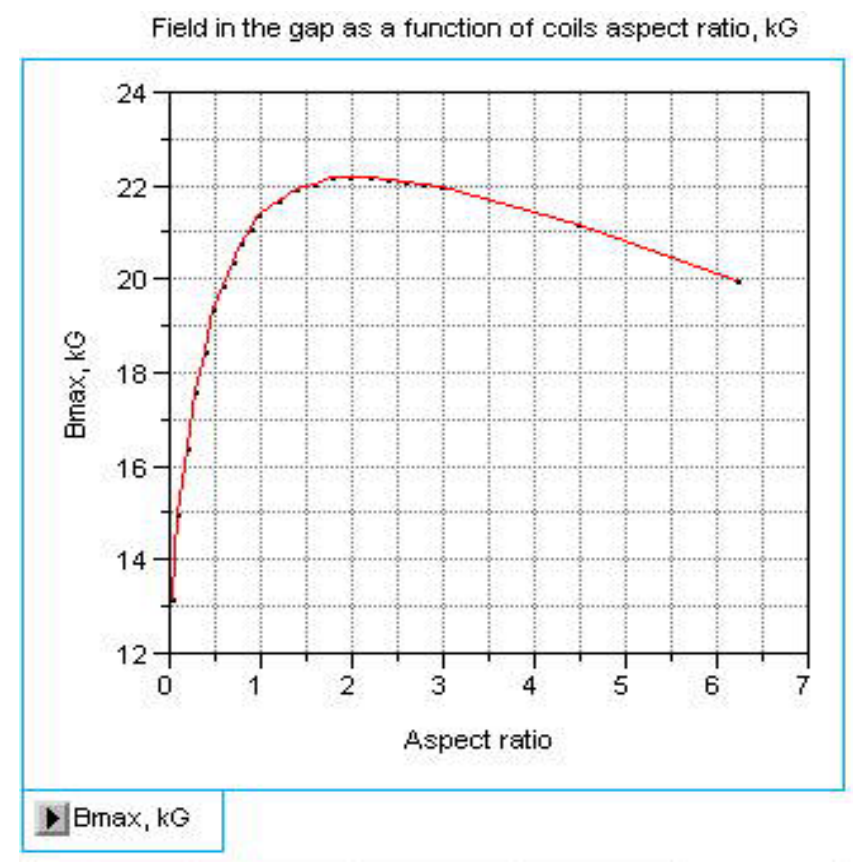

FIGURE 3: Field is the gap between poles as function of the width/height cross-section ratio (Fig.1) for the coil [2]. Flat coils are at the right. Cylindrical ones-- at the left.

It is clear that the wires located far from the medial plane give exponentially small input into the field at medial plane. This is physical explanation of the shape of curve in Fig.3.

One can see from this Figure, that maximal field corresponds to the coil which width is twice the height. Finally the coil cross section was chosen with cross section $\cong 1^{\prime \prime} \times 0.75^{\prime \prime}$ for simplicity of winding.

Evolution of temperature inside the coil defined by parabolic equation

$$
\kappa \frac{\partial^{2} T}{\partial x^{2}}=\rho c_{v} \frac{\partial T}{\partial t}
$$

where $\boldsymbol{\kappa}$ is a heat conductance, $\rho$ is density of the coil, $c_{v}$ is a heat capacity. Here $x$ marks coordinate, rectangular to the wire. Equation (8) defines heat-skin layer depth $\delta$ as

$$
\delta^{2} \cong \frac{\kappa}{\rho c_{v}} \tau
$$

where $\tau$ is a time constant. Substitute here averaged over cross section copper/epoxy parameters one can obtain the time relaxation constant for a $\delta=1 \mathrm{~cm}$ thickness as $\tau \sim 100 \mathrm{~ms}$, mostly defined by parameters of epoxy.

In Fig.4 the curve shown which describes the field in the gap as function of thickness of steel plate, caring the poles. This is important for minimization of the yoke weight. The height was chosen equal to $\cong 14.5 \mathrm{~cm}$. It is slightly below the saturation, but saved about one inch of steel, lowering field on $\sim 5 \%$ only. 


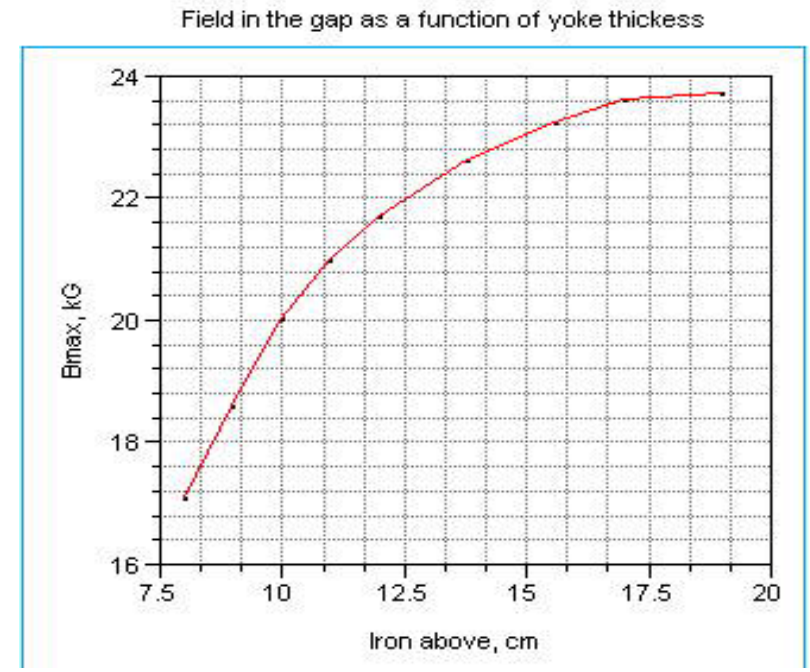

FIGURE 4: Field is the gap between poles as a function of the iron height [2].

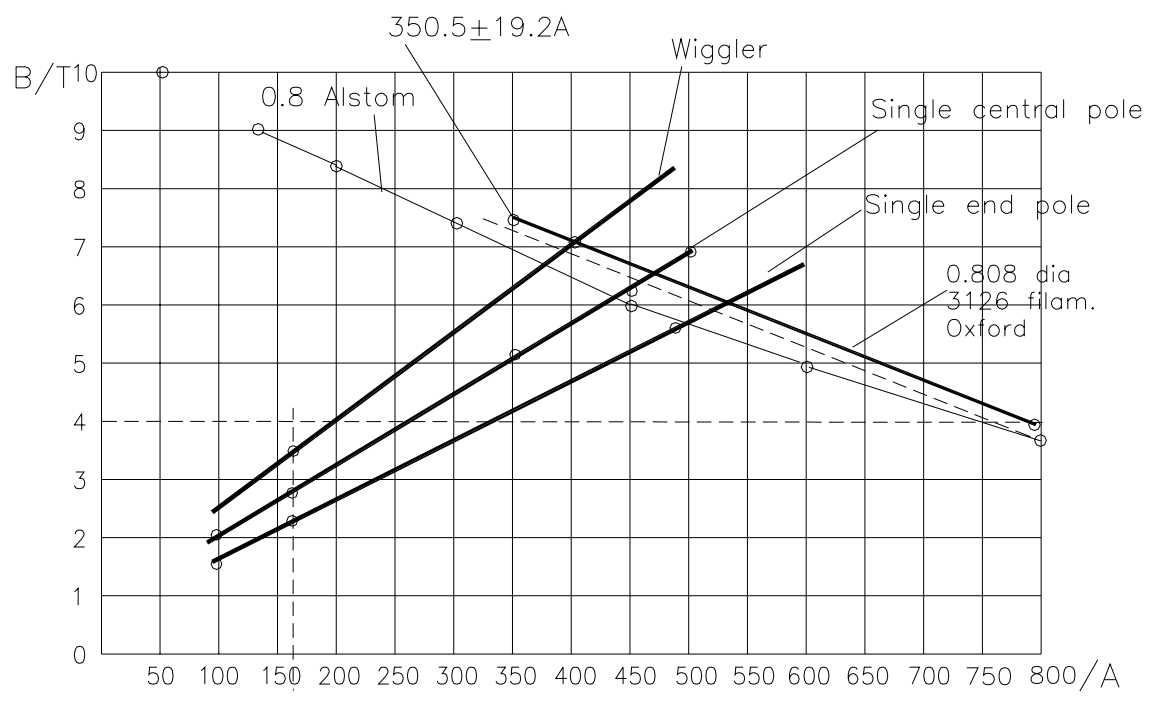

FIGURE 5: The loading curves.

Loading curve is shown in Fig.5. There are also curves for single poles. These required for test of single poles in Dewar. The operating current 161 A; 141A for new wire with 660 turns, is about half of the critical current defined at crossing the loading curve with characteristic curve of wire. The margins were chosen intentionally so wide to avoid any problem with training and quenching in a future. All present experience confirmed that this choice was right.

Technology is described in [7]. As it can be seen from Fig. 1 the magnet cold mass is a steel plate with poles attached to it. Coil winding and curing were done directly on the pole. Thin G10 insulation wraps the kern. The coil wounded in few layers at a time. Each of these layers formed by $\sim 21$ wires impregnated by Epo-Tek T905 Epoxy. The paper NOMEX was used for interlayer insulation. After every four layers of windings, the coil restrained for curing during $\sim 1.5$ hours with the side pressure applied up to $\sim 30 \mathrm{~atm}$ on it. During the winding the tension applied to the wire was at the level $\sim 12 \mathrm{lb}$. per wire diameter $d \cong 0.8 \mathrm{~mm}$. Teflon spray allowed us to avoid sticking between the parts [7]. 
Splitting the coils into the layers defined by peculiarity, that after curing under compression every last layer, all inner layers becomes squeezed at room temperature by outer ones. This prevents the coil strength degradation after cooling to the Helium temperature. The very last outer layer is located in places, where magnetic field is about zero, so there is no danger for triggering quench.

To prevent coil destruction initiated by quench, passive resistors attached to each coil was suggested [2] as simplest solution.

\section{TAPERING}

Tapering, or variation in field strength along the wiggler, used when there is a desire to have zero (or any) average displacement of trajectory in a wiggler. One can see that this procedure does not change the amplitude of wiggling as it is defined by magnetic field and period of wiggler. For even pole wiggler situation is different however.

Although it is likely evident, it was mentioned first in [8] however, that end poles with fields integrals $1 / 4,-3 / 4,+1$ of regular one gives zero displacement of trajectory. If periods are the same these numbers reflect the field strengths at each pole.

This is not the only solution, however. Let us consider the series of odd numbers with reversing signs as

$$
1,-3,+5,-7,+9, \ldots(-1)^{k}(2 k-1), k=0,1,2,3, \ldots
$$

Now if we brake this sequence at any $k=m$ then the sum of these terms goes to be

$$
1-3+5-7+9-\ldots(-1)^{m}(2 m+1)=\sum_{k=0}^{m}(-1)^{k}(2 k+1)=(-1)^{m}(1+m) .
$$

Let these numbers will represent now the nominators of fractions of the field integrals for each pole normalized to the maximal one in regular part of wiggler. So the next pole after $m$, supposed to be a main one, must have the value counted as $(2 m+1)+1$, just one unit more, and opposite sign. Now one can obtain, that full integral over all wiggler will be

$$
\begin{aligned}
& \mathrm{S}(m)=1-3+5-\ldots(-1)^{m}(2 m+1)+(-1)^{m}(2 m+2)+(-1)^{m}(2 m+1)+\ldots+5-3+1= \\
& \quad=2 \sum_{k=0}^{m}(-1)^{k}(2 k+1)-(-1)^{m}(2 m+2)=(-1)^{m} 2(1+m)-(-1)^{m}(2 m+2) \equiv 0 .
\end{aligned}
$$

One can also find that $\sum_{m=1}^{L} S(m) \equiv 0$. This means that the field first and second integrals over all wiggler are zero. So the recipe is very simple. One chooses the number of tapered poles and makes the side pole integrals proportionally increasing odd number each starting from the end until it comes to desirable number. The main pole integral must have the value one unit more than the last number in series and opposite sign. After that the pole strengths must go in descending order. One can add in central part any number of pairs of central pole with reversing signs.

For example series as $+1 / 8,-3 / 8,+5 / 8,-7 / 8,+1$, $[\mathrm{n}(-1,+1)],-7 / 8,+5 / 8,-3 / 8,+1 / 8$, where fractions stand normalized to central pole integral, will also give zero angle and displacement. Here was chosen the last number in series (1) to be -7 , central pole must have integral equal to 8 and any number of poles with $(-8+8)$ pairs can be added at central region. One can easily find the sequence of tapering for $6,8,10,12,14,16, \ldots$, Fig. 6 , and so on poles. 
We called this adiabatic entrance.

Once particle entered into wiggler with such tapering and began its oscillations following sinusoidal trajectory, there are two ways out. First is a symmetrical exit, when the sign of deflection in the last pole is the same as during entrance, and the second one is asymmetric one, when the kick is going in opposite direction. In our sequence this can be arrange by adding one more pole of next following sign and reversing the signs of descending poles at other side. So the polarity of each pole symmetrically located from center point, which is now between poles, are opposite. Obviously in first case the total number of poles is odd, in second case it is even.

Some times tapering is extremely useful allowing any law in establishing equilibrium. This is especially important for undulators, when $\rho / \gamma>\lambda_{u} / K$, where $\rho$ is a bending radius in the field of pole, $\lambda_{u}$ is period of undulator, $K=e B_{y} \lambda_{w} / 2 \pi m c^{2}=B_{y} \lambda_{w} \gamma /(H R)$, allowing narrowing spectrum. Amplitude of oscillation remains the same, however, $a \cong \lambda_{w} K / \gamma$.

In last millennium it was very popular to carry wiggler measurements with long coils. Some authors recommended make poles narrow, as all non-linearity are compensated in alternative sequence. That is why real properties of wiggler were understood only recently. Really the first

integral $I_{1}(x, y, s)=\int_{-\infty}^{s} B(x, y, \sigma) d \sigma$ and second integral $I_{2}(x, y, s)=\int_{-\infty}^{s} I_{1}(x, y, \sigma) d \sigma$, where $\sigma$ is running along the wiggling trajectory, define the wiggler properties. If trajectory taken as a line, like long coil models it, these integrals reflect the properties in general only. Full picture can be obtained by tracking through real fields of wiggler. For wiggler with odd number of poles second integral is proportional to the first one, so it is easily to find any indications of missed perfection even by a long coil. But this will be only indication, as the long coil does not reflect wiggling of trajectory. For even-pole wiggler this will not give any indications at all. Measuring with long coils in the wiggler, especially even-pole one, is able to find brutal mistakes only.

One disadvantage associated with tapering is a necessity to have few different types of coils. This definitely increases the cost of fabrication. However adiabatic entrance is useful for helical undulators having very short period for example, as exact managing the pole strength at the end is not an easy task.

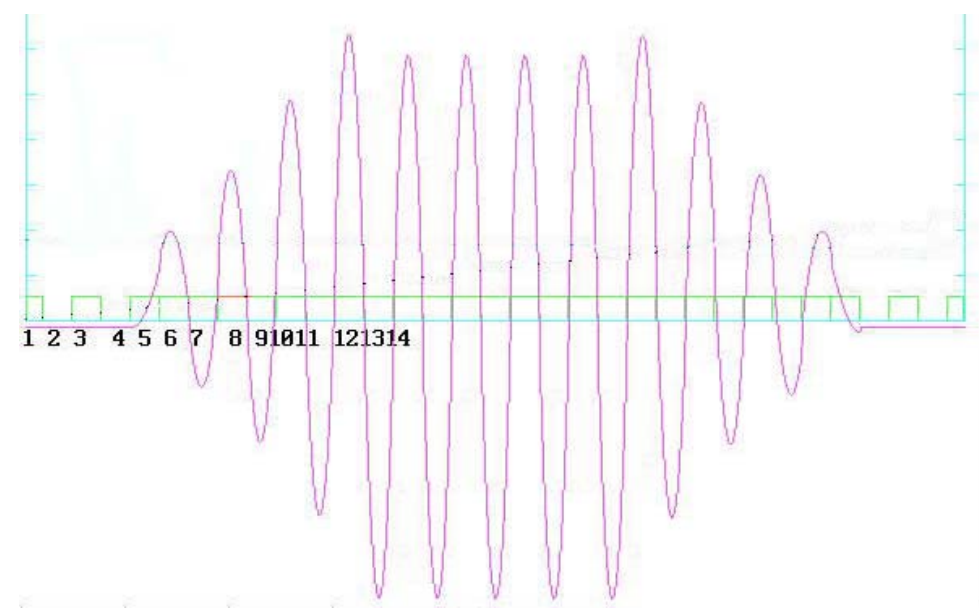

FIGURE 6: Trajectory of particle in a wiggler with tapering $\sim 1 / 16,-3 / 16,+5 / 16,-7 / 16,+9 / 16,-$ $11 / 16,+13 / 16,-15 / 16,+1$. Doublet of quadrupoles envelops wiggler from each side. Same period. 
Seven-pole wiggler was made with tapering 1/2, -1, Fig.7. This gives displacement $\Delta \cong \lambda_{w} K / \gamma$ what is amplitude of wiggling but this, first, supposed to reduce the coil types to two only and to have maximal period for reduction of nonlinearities.

For eight-pole wiggler tapering $1 / 4,3 / 4,1$ is the only way, practically, as trajectory amplitude $a \cong \lambda_{w} K / \gamma \cong 1.5 \mathrm{~mm}$, and doubling this value may yield significant nonlinear effects in deflection. Trajectories in eight-pole wiggler represented in Fi.8 for comparison.

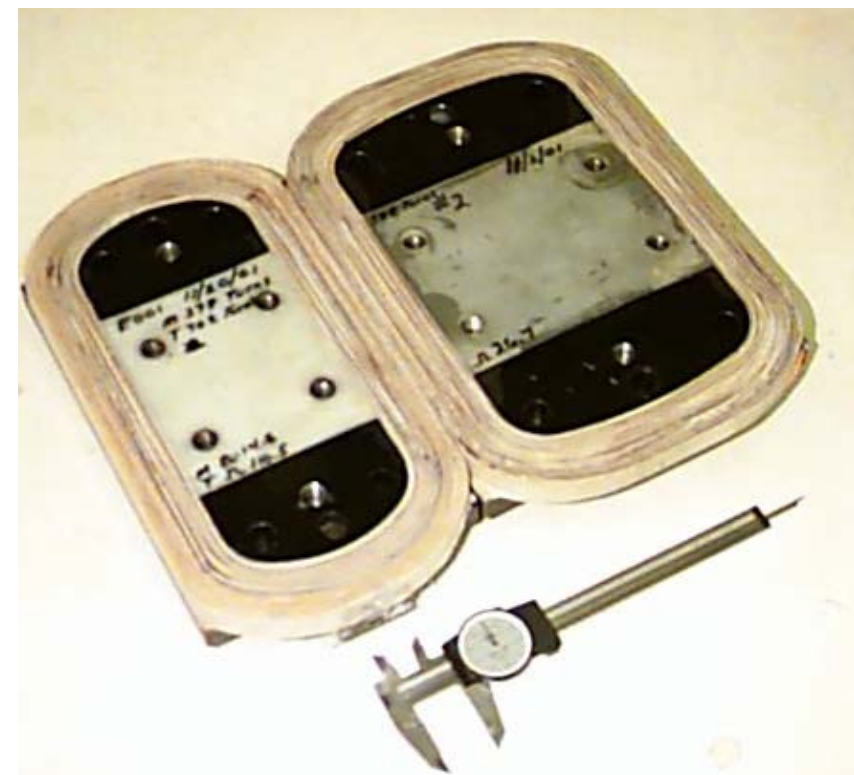

FIGURE 7: Two poles: $20 \mathrm{~cm}$ and $15 \mathrm{~cm}$ with coils shown together. G10 plate covers the lowering, see Chapter 4.
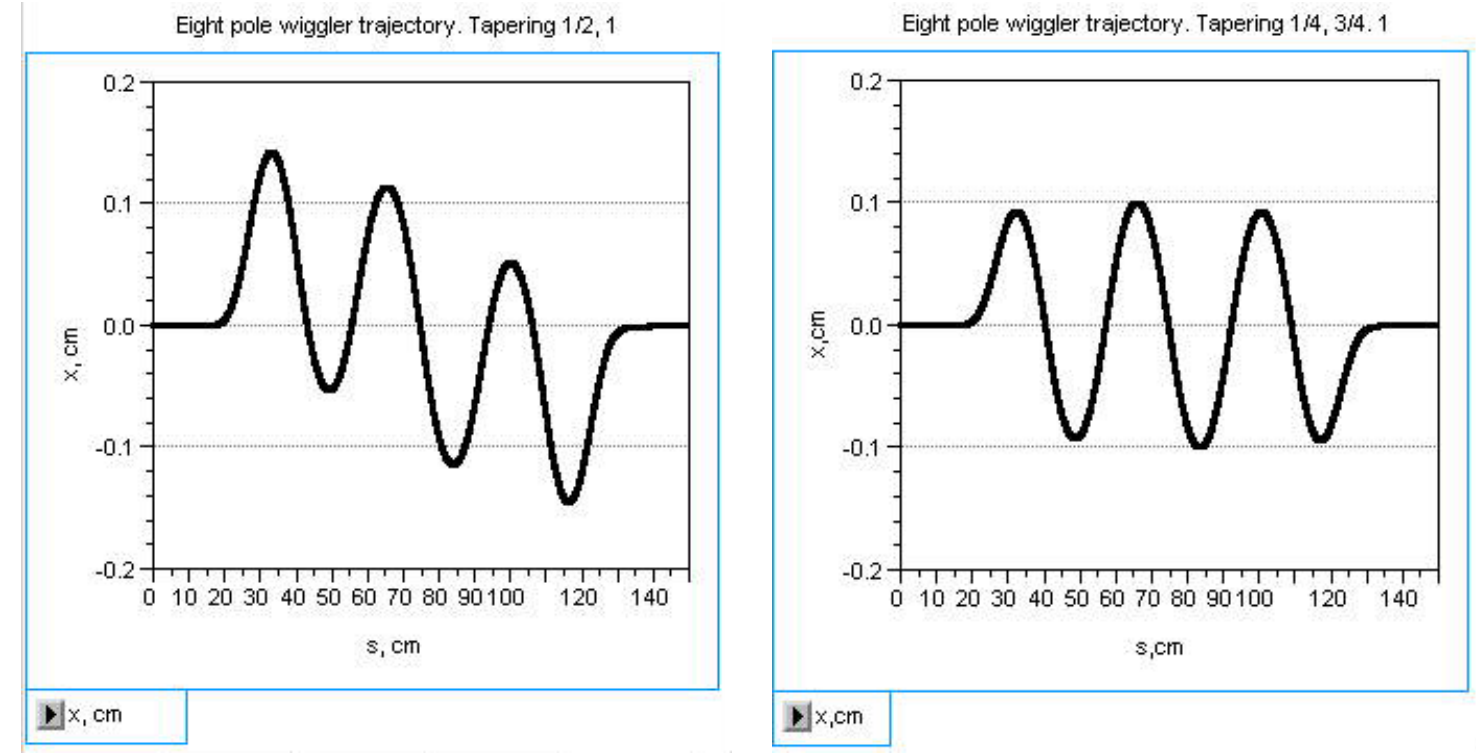

FIGURE 8: Examples of trajectories in 8-pole wiggler with different tapering. At the left - tapering is $1 / 2,-1,+1, . .1 / 2$ made with $10 \mathrm{~cm}$ pole, next pole is $15 \mathrm{~cm}$ with added wires, next is a regular $20 \mathrm{~cm}$ one. At the righttattering is $1 / 4,-3 / 4,1$. Same iron for the poles used, but different wiring. 
For this particular ratio of iron widths, the amplitude sweep at the left is 1.5 times bigger, than for the right. For the poles with same period this ratio goes to 2. Tapering in 7-pole wiggler as example is shown in Fig.9.
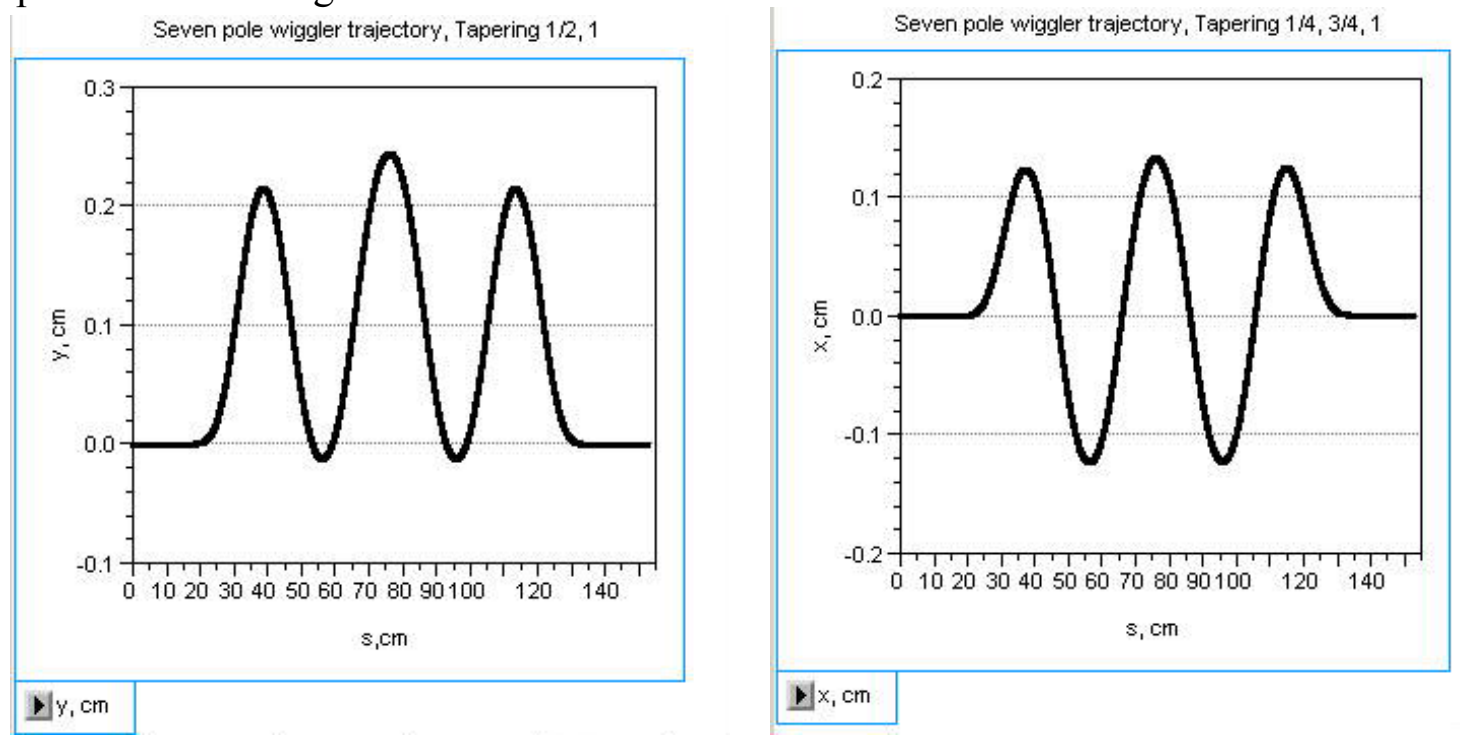

FIGURE 9: Examples of trajectories in 7-pole wiggler with different tapering. At the left -tapering is $1 / 2,-1,+1, \ldots 1 / 2$ made with the same iron as in Fig.7. At the right- tattering is $1 / 4,-3 / 4,1$. Same iron for the poles used, but different wiring.

Knowing the fields and having tracking code in hand one can investigate properties of wiggler model dynamically. For example, for seven-pole wiggler, in Fig. 10 there are shown integrals along straight lines, as they can be measured by long coil. One can see that at design field level 1.7 T the line is mostly flat. Here in this figure only half of graph is shown; graphs for $x<0$ are symmetric with respect to the ordinate axes.

Integrals along straight lines, 7 pole, $\mathrm{kGcm}$

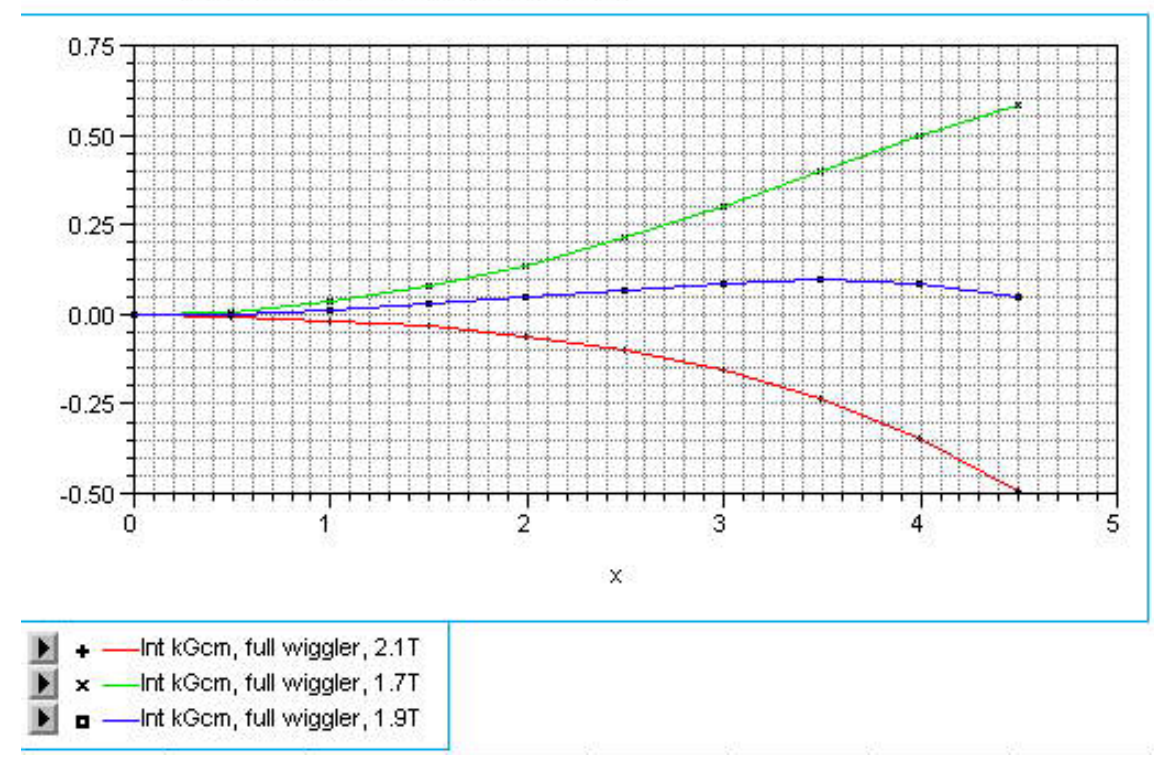

FIGURE 10: (Color) Integrals along straight lines for different feeding currents. 
Kicks, obtained by particles running across aperture with different $x$ - coordinate at the entrance is shown in Fig.11. All kicks remain in $\pm 50 \mu \mathrm{rad}$ for all fields and not correlated with integrals in Fig 10.

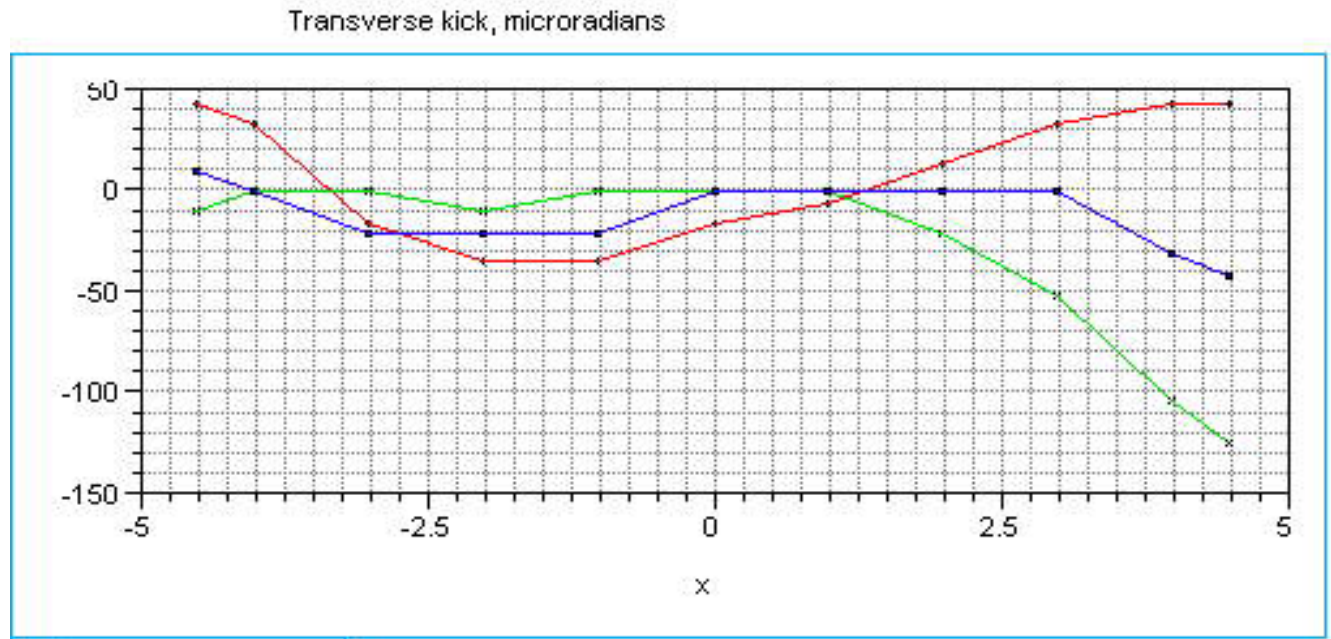

$$
\begin{aligned}
& + \pm-X^{\prime} 7 \text { pole2.1T } \\
& \mathbf{x}-\mathrm{X}^{\prime} 7 \text { pole1.7T } \\
& \mathbf{a}-\mathrm{X}^{\prime} 1.9 \mathrm{~T}
\end{aligned}
$$

FIGURE 11: (Color) Horizontal kicks, $\mu \mathrm{rad}$, as functions of transverse input offset, $\mathrm{cm}$, for different fields in a 7 pole wiggler.

In Fig.12 the graphics show the difference between input and output coordinates for 7-pole wiggler for different fields in a wiggler.

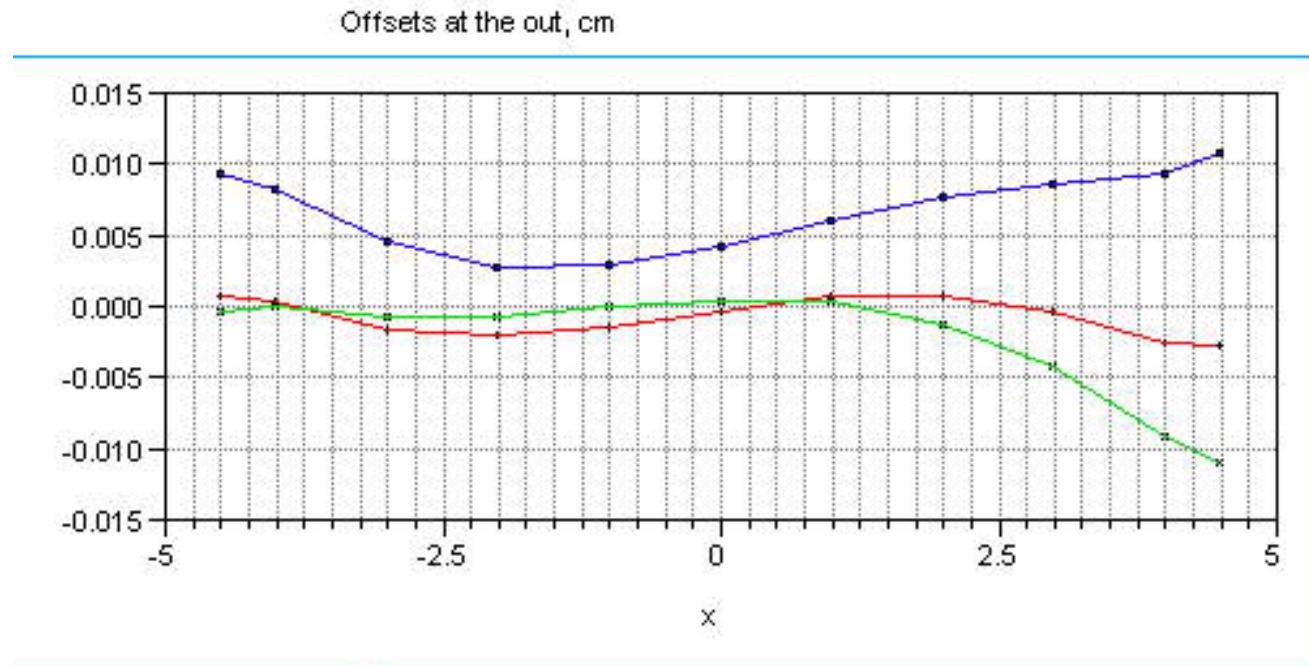

$$
\begin{aligned}
& +-\times 71.9 \text { out }-x 0 \\
& \times-\times 71.7 \text { out } \mathrm{x} 0 \\
& \mathbf{x}-\mathrm{x} 2.1 \text { out } \mathrm{x} 0
\end{aligned}
$$

FIGURE 12: (Color) Differences between output and input horizontal coordinates in 7-pole wiggler. Nonzero displacement can be eliminated by trim coil. 


\section{SHIMMING}

The field roll off from the wiggler center provokes nonlinear effects, as when particle wiggles with significant amplitude $\sim 1.5 \mathrm{~mm}$ the nonlinear terms from neighboring poles are not compensating each other, especially with pretzel-like trajectory. To reduce this effect the pole top field must be as flat as possible. The pole with the width much more than the wiggler period is a good way to solve this problem. The old fashion way as shimming is also working here, despite the iron is deeply saturated.

Poles having length $20 \mathrm{~cm}, 15 \mathrm{~cm}$ and $10 \mathrm{~cm}$ were developed during these years, Fig. 14. The $10 \mathrm{~cm}$ poles successfully used in 3-pole model [7] and are in use in 8-pole one.

Width of the deeps was chosen as a half of all pole width and with simplest shape.

Suggested operational margins, as we mentioned above, for the wiggler fields are 17-21 kG. So the wiggler field optimized for the middle of this diapason, namely $19 \mathrm{kG}$.

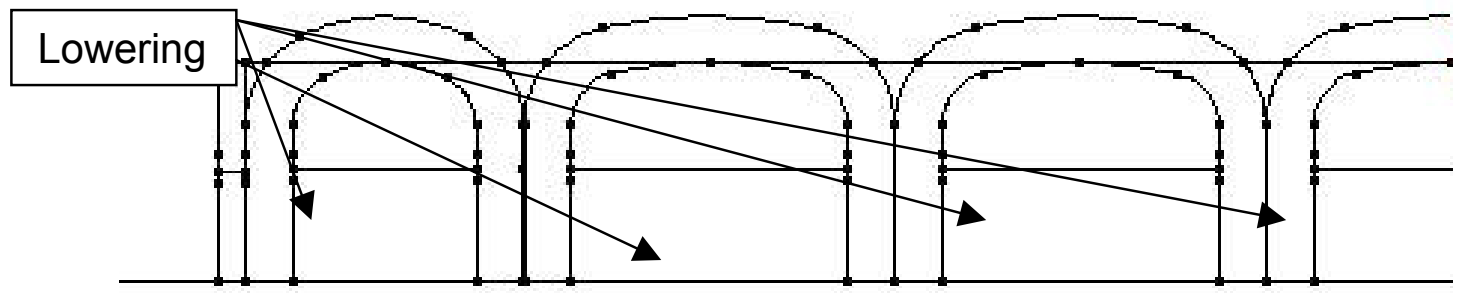

FIGURE 13: Top view on the wiggler poles. Half of the 7-pole wiggler is shown. The main poles lowering are 5 $\mathrm{mm}$.

Single plane lowering was chosen for simplicity, more complex types as one side chamfer might be more effective, but requires more efforts for fabrication.

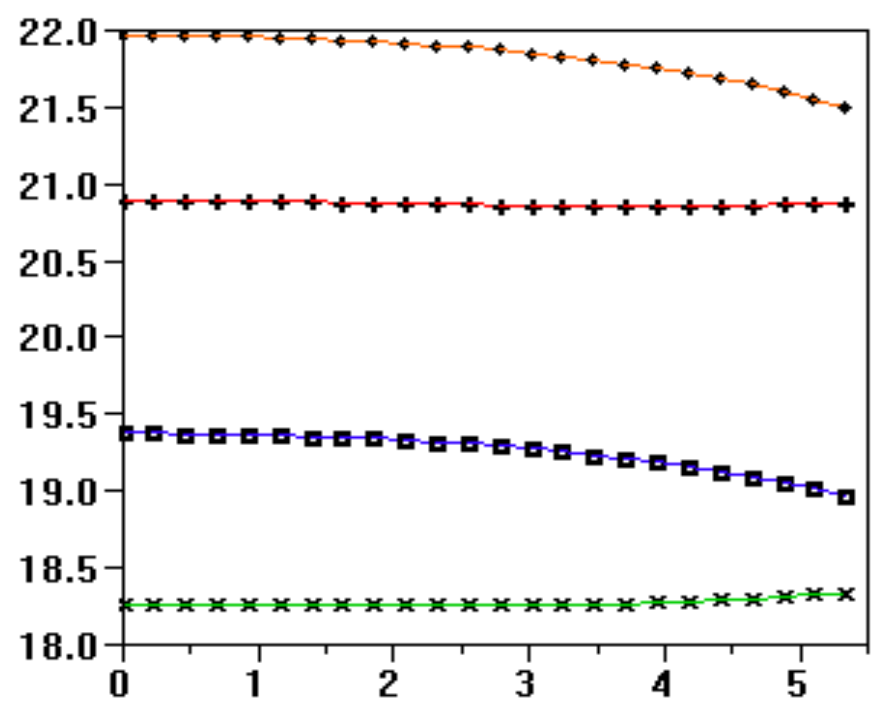

FIGURE 14: Vertical field, $k G$ as a function of transverse coordinate $x, \mathrm{~cm}$ for two different feeding currents and pole shapes-flat and with lowering. Vacuum chamber ends at $\pm 4.5 \mathrm{~cm}$. 

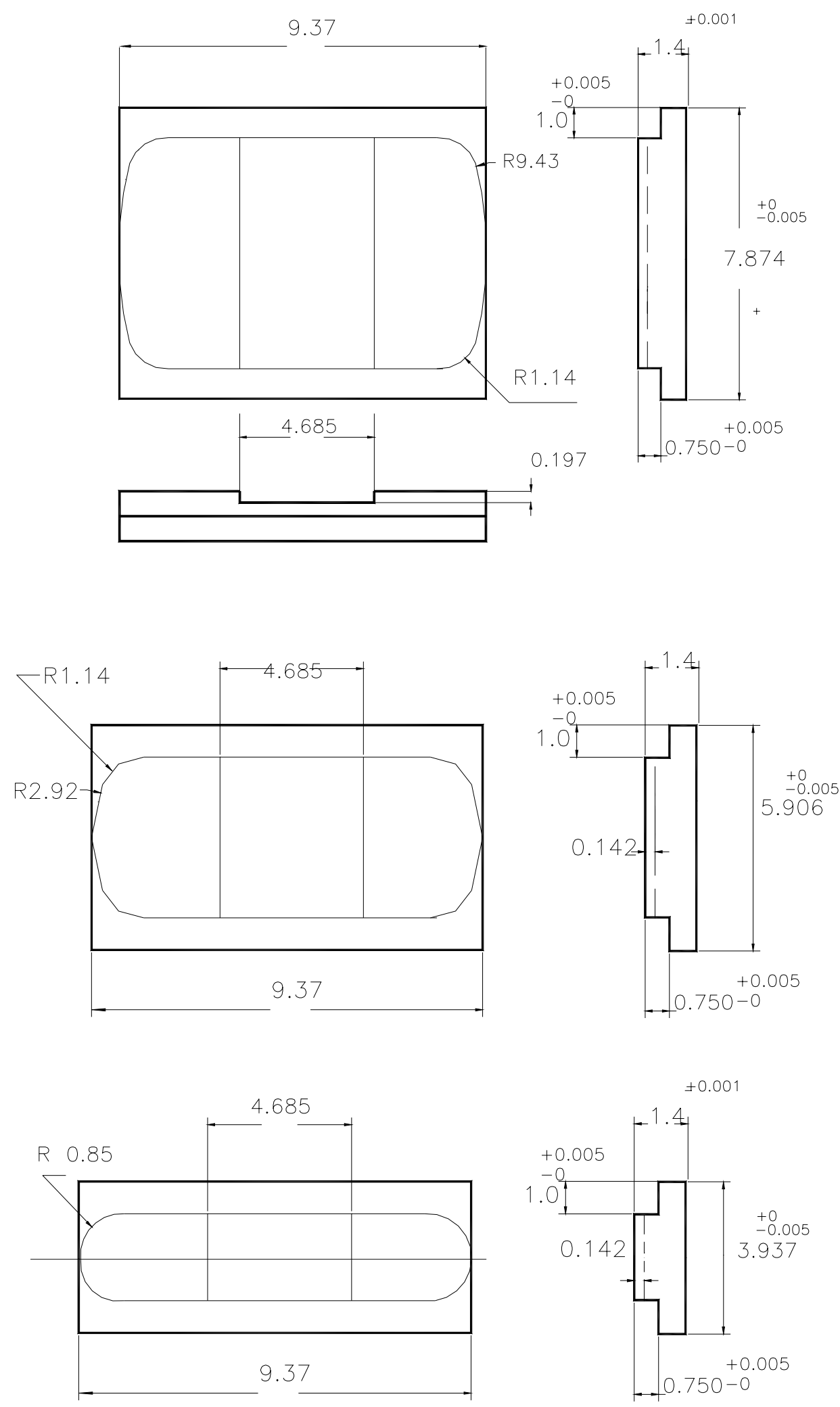

FIGURE 15: $20 \mathrm{~cm}, 15$ and $10 \mathrm{~cm}$ poles developed for wigglers. 


\section{POLE FACE WINDINGS}

Next step in flattering is active correction. Next allowable multipole in transverse expansion is sextupole. So if one puts a coil on the pole surface it will generate the field with the same symmetry, i.e. dipole, sextupole,... . So as adjusting main current can neutralize the dipole field change, this coil can be considered as a source of sextupole. Lowering at the center is an ideal place for positioning this correction coil.

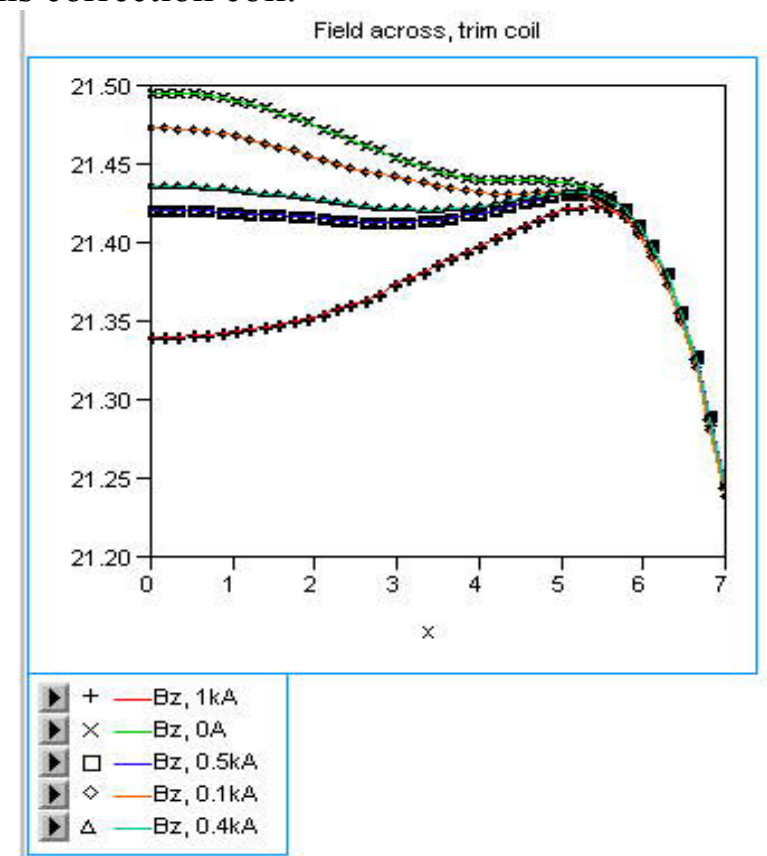

FIGURE 16: The field across the pole as a function of current in trim coil. Main feeding current is $95 \mathrm{kA} /$ pole. Material of the pole is annealed Steel 1010.

For winding the SC wire with 0.017 " diameter was used. This dimension includes Capton tape wrapping and Bonadll impregnation. After winding form with coil was heated $\sim 200^{\circ} \mathrm{C}$ and after cool down a solid coil obtained.

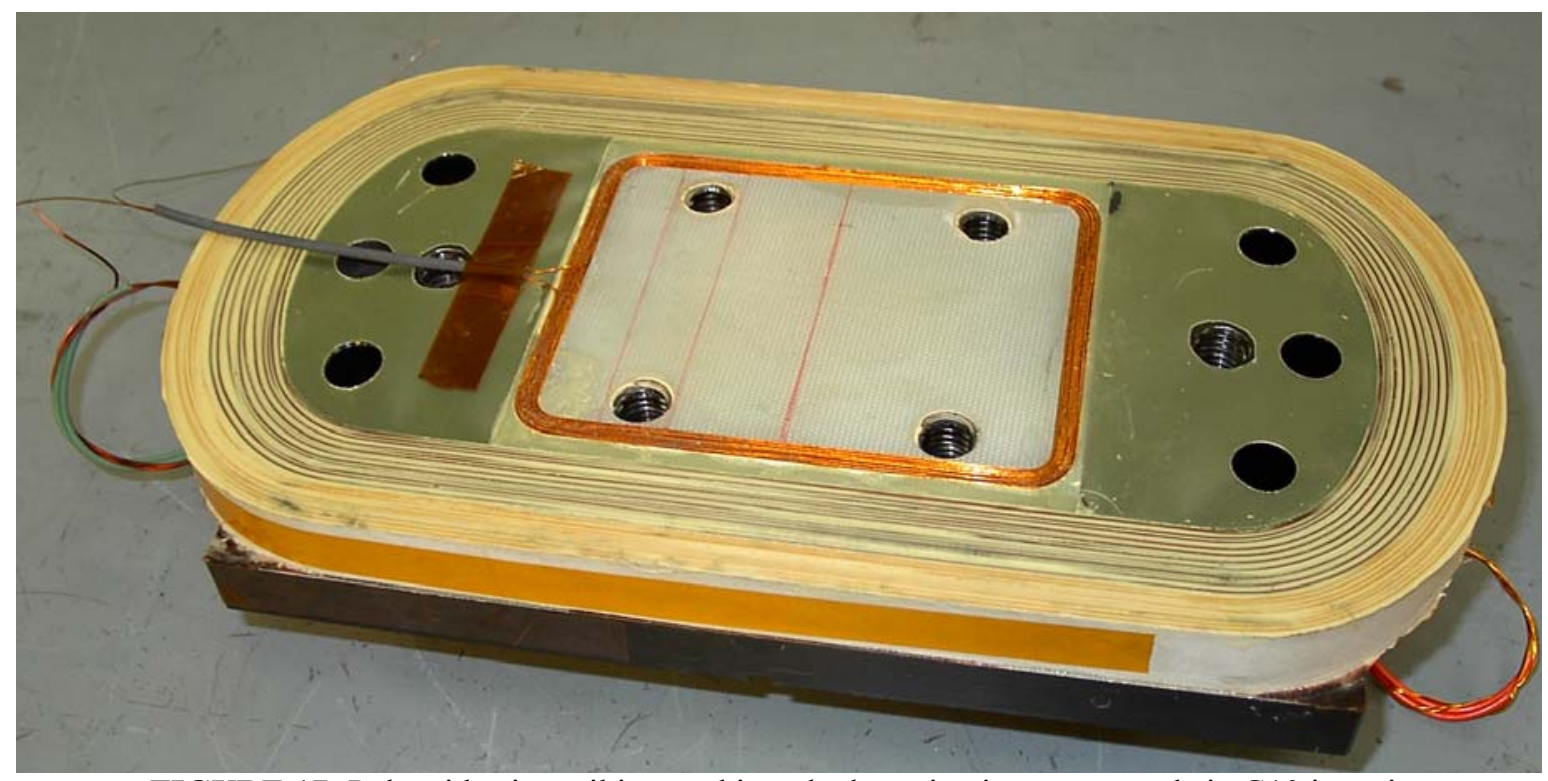

FIGURE 17: Pole with trim coil inserted into the lowering in groove made in G10 insertion. 


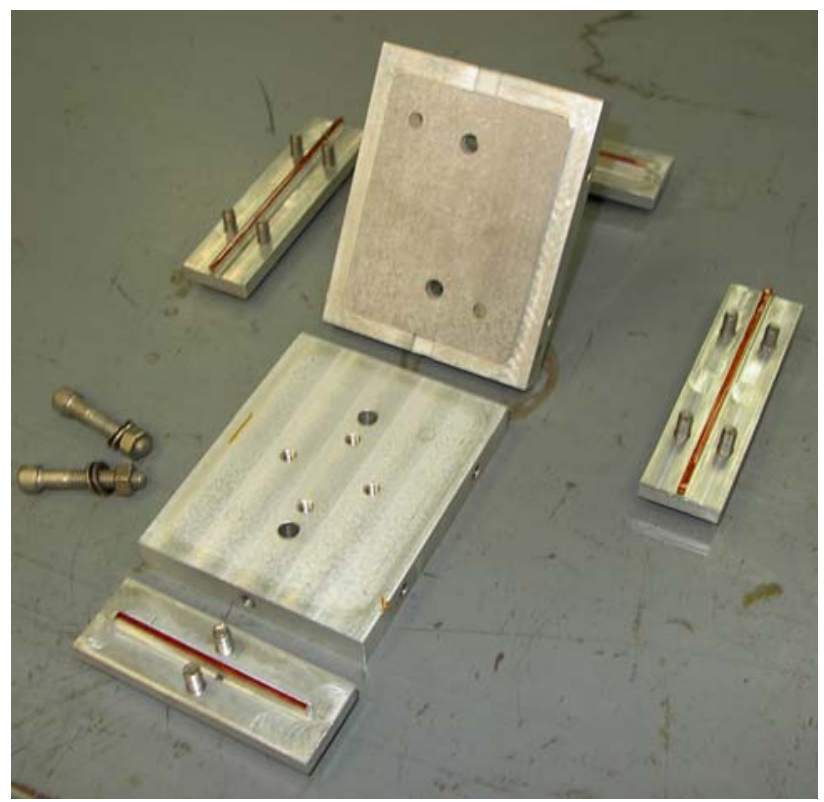

FIGURE 18: The winding form for trim coil.

The coil having 100 turns will require the feeding current of $400 / 100=4$ A only. At the lower edge of operational limit fields the sign is opposite. At the field level $\sim 19 \mathrm{kG}$ the current is about zero. The coil enclosed into groove milled in G10 plate, which covers the lowering at the pole center.

Poles with coils wounded and cured on the pole attached to the steel plate (Fig.1) enveloped by stainless steel cover. Upper and lower halves have own envelope, Fig. 19 [2]. In Fig. 20 the photo of the two joined halves is shown. StSteel cover not welded yet to the stainless steel base.

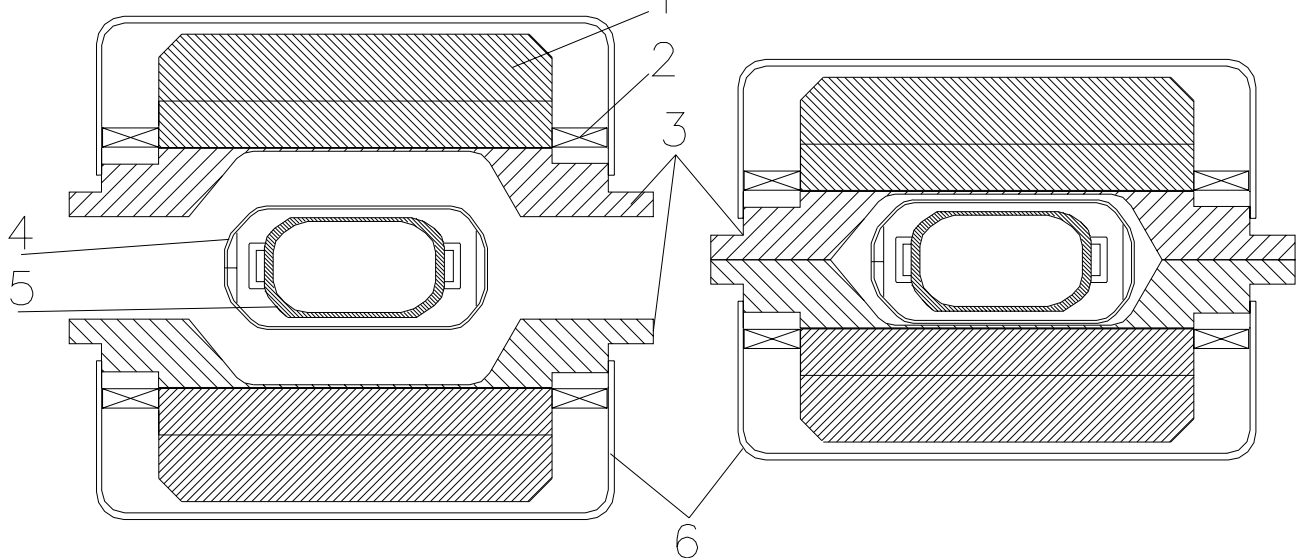

FIGURE 19: The wiggler's cold mass cross section concept [2]. 1 is a soft steel plate with poles, 2 are the coils, 3 -StSteel caring plates, 4- LNitrogen screen, 5 -room temperature copper chamber. Room temperature aperture is $9 \times 5 \mathrm{~cm}^{2}$.

Dense hatch in Fig. 19 marked as 1, related to the soft iron, rare, 3 -to Stainless Steel. Iron begins at $1.5 \mathrm{in}$ from median plane. Upper and lower containers for liquid Helium are connected through the side tubing. This design drastically simplifies assembling. 


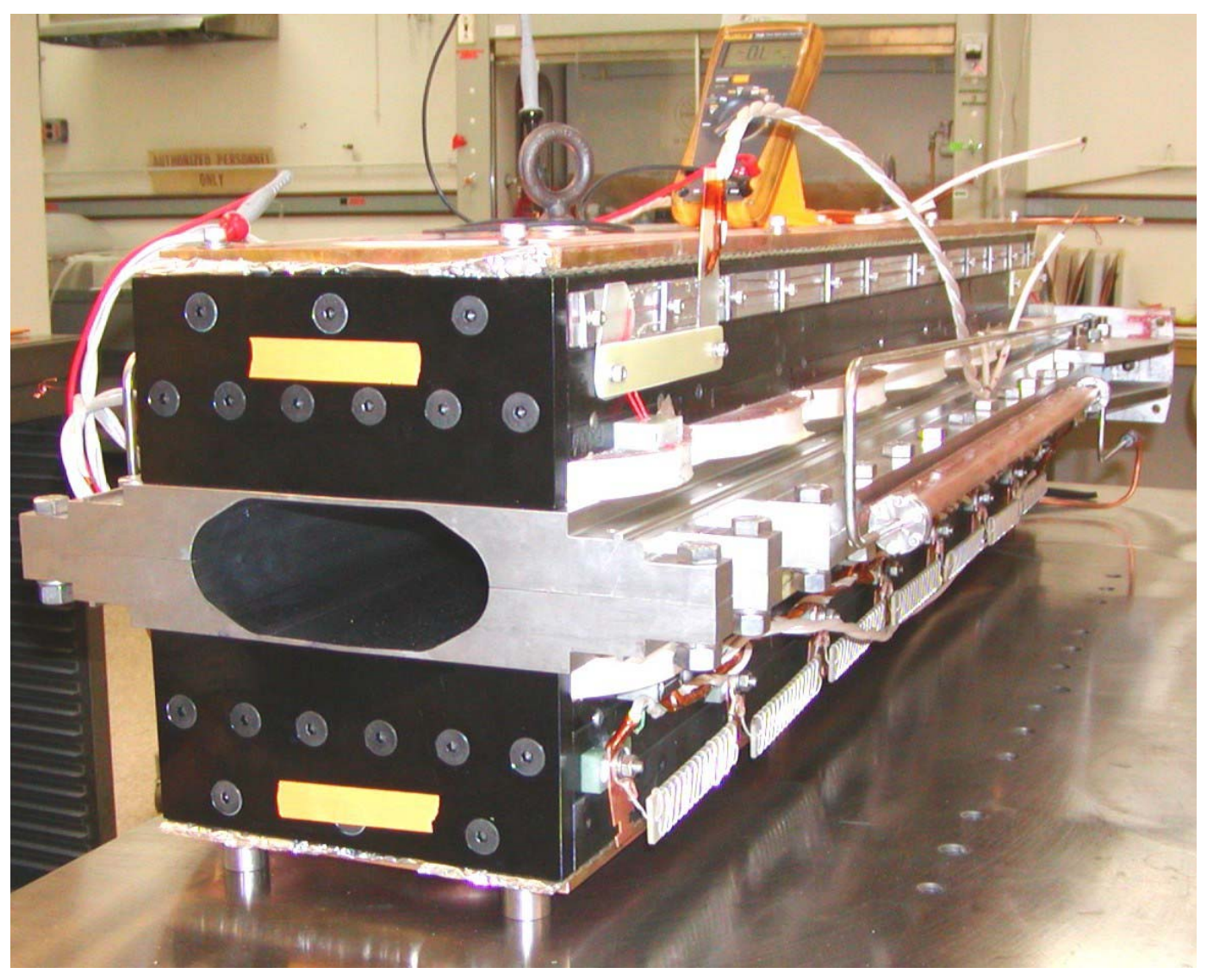

FIGURE 20: Cold mass photo. Realization of principle shown in Fig.19, Stainless steel cover is not welded around yet.

\begin{tabular}{|c|c|}
\hline Parameter & Value \\
\hline Height of the cold iron & $29 \mathrm{~cm}$ \\
\hline Pole length & $20 / 15 \mathrm{~cm}($ end pole $)$ \\
\hline Number of poles & $5+2,4+4$ \\
\hline Current & $150-165 \mathrm{~A}, 141 \mathrm{~A}$ \\
\hline Pole width & $23.8 \mathrm{~cm}$ \\
\hline Field & $1.7-2.1 T$ \\
\hline $\begin{array}{c}\text { Vertical cubic } / \text { linear kick } \\
\text { ratio }\end{array}$ & $\delta \leq 8 \% @ 2 \mathrm{~cm}$ in $|x| \leq 5 \mathrm{~cm}$ \\
\hline Energy stored & $\sim 47.3 \mathrm{~kJ}($ at $2.2 \mathrm{~T})$ \\
\hline
\end{tabular}

\section{MEASUREMENTS}

To the moment six wigglers total were measured so far. Two of theses have seven-pole structure, four eight-pole ones. Measurements were carried with Hall probe device F.W.Bell 6010 series. Thermo-stabilized probe can be inserted into fixed transverse positions in a cartridge, which can be carried by stepping motor along the wiggler, Fig.21. Special measurements made at the beginning and at the end of $\sim 8$ hours session, showed, that the difference in readings at the same 
points are less that 5G. Reversing Hall probe showed, that the readings stay within $\leq 1.25 \cdot 10^{-3}$ for all readings. As we made comparison with central line, this accuracy is fine.

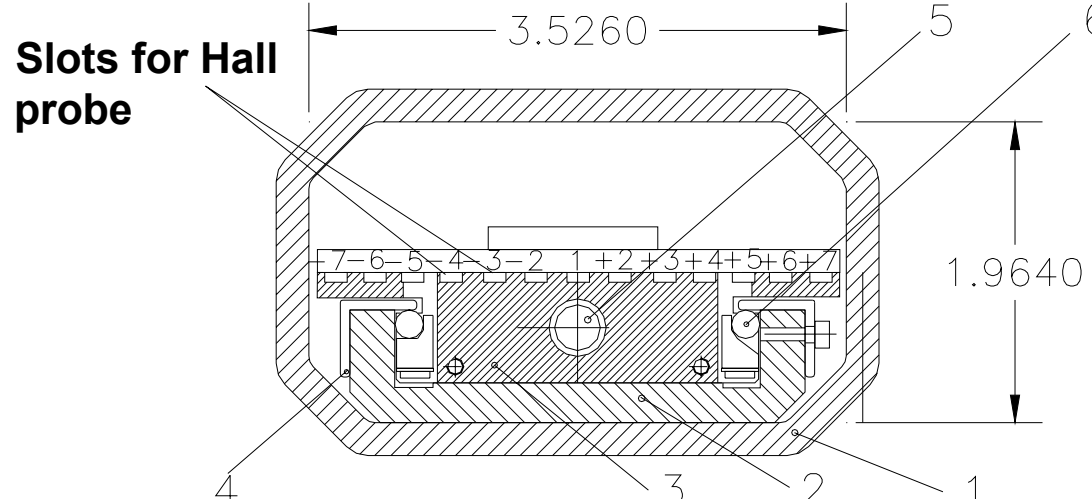

FIGURE 21: Hall probe cartridge assembly cross-section. 1 is the warm copper vacuum chamber with dimensions in inches, 2 is aluminum rail, 3 is a cartrige with slots for Hall probe, 4 is holding profile, 5 is a shaft with $1 / 16$ " thread, 6 represent alignment fixtures. Slots are numbered as they appear in the file descriptor.

Number of longitudinal points is 1060, going through 1/16". Signal acquired at every point becomes written to the file (having 1060 data rows). Each of these files marked in association with transverse position of Hall probe in cartridge, direction of motion of the cartridge, data, when taken. Slots in cartridge have transverse period $0.625 \mathrm{~cm}$, so mostly deflected from center ones running at \pm 3.75 off central line.
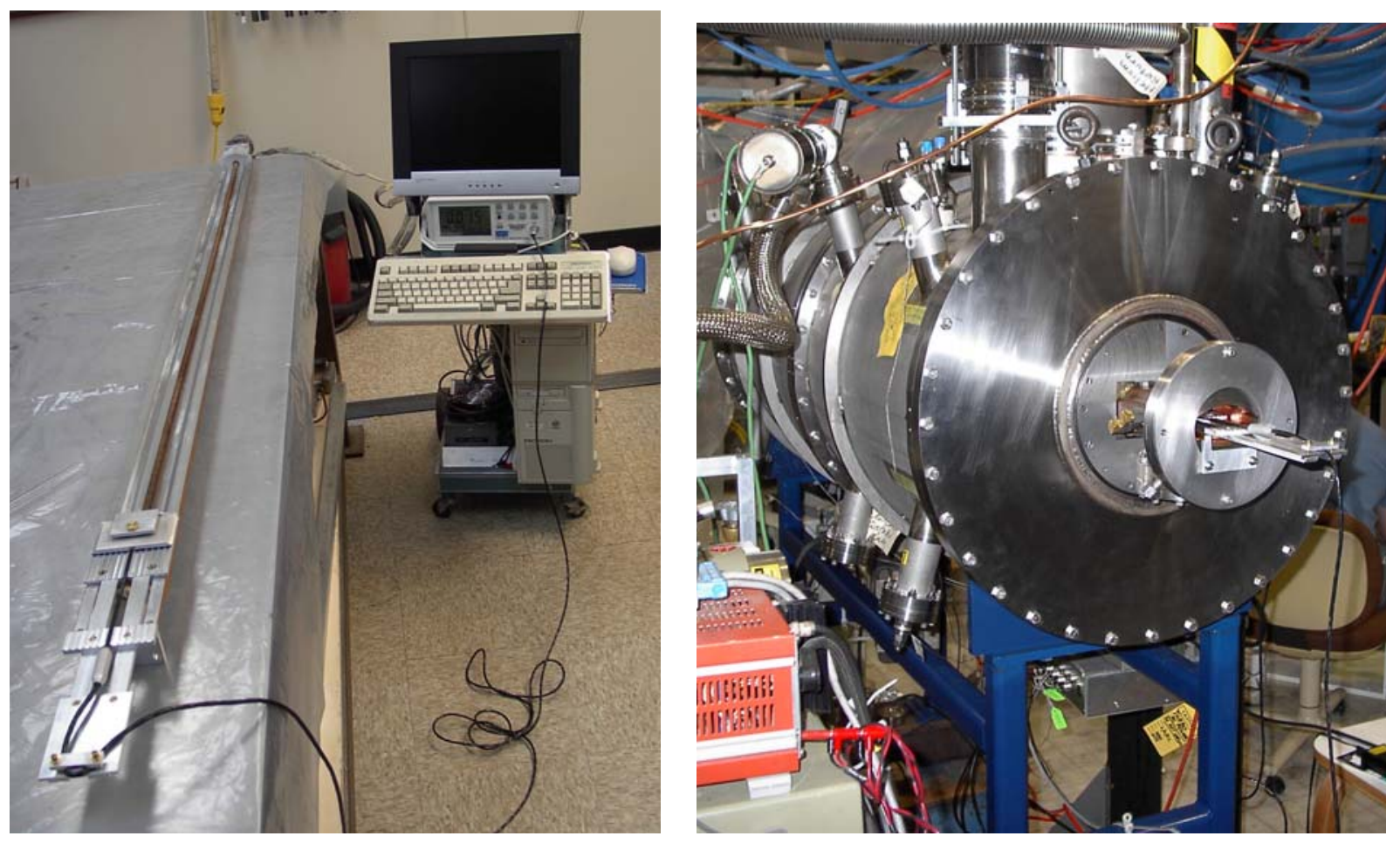

FIGURE 22: Left- Cartrige with Hall probe installed on the rail. Right-The rail inserted in vacuum chamber. 

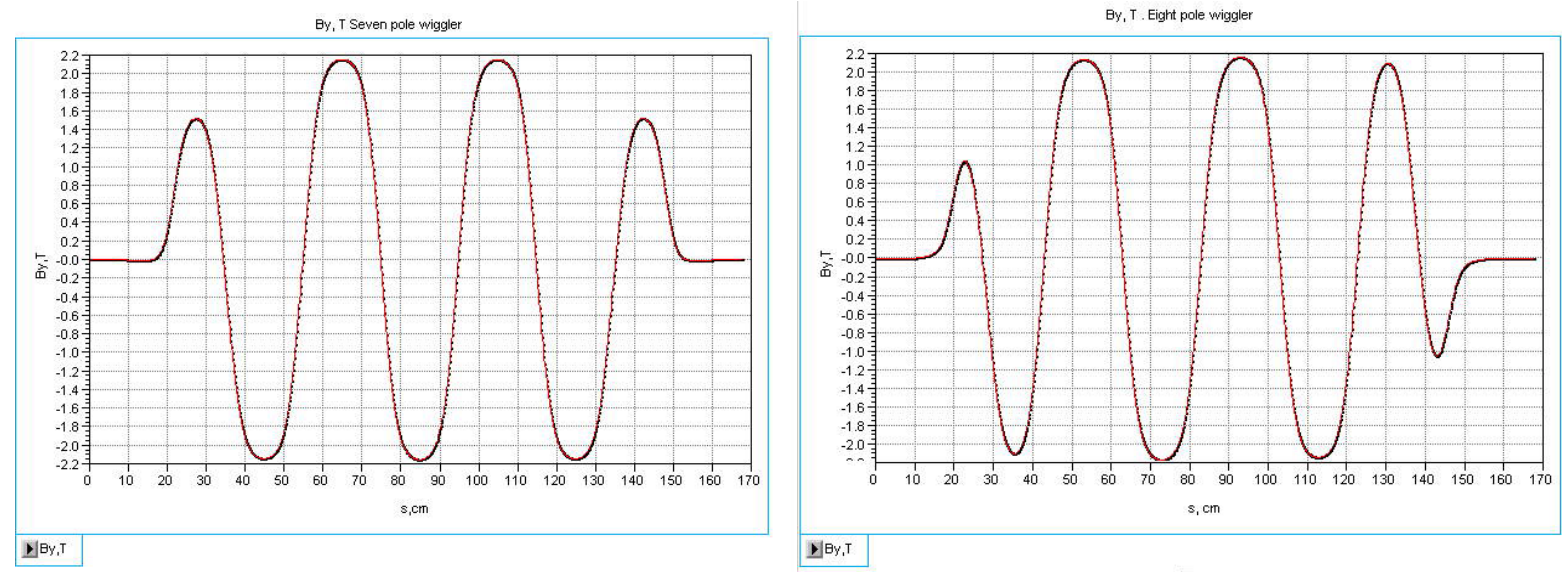

FIGURE 23: Measured longitudinal distribution for seven and eight pole wigglers.

Calculated fields were compared with many files containing measured data.

One can see from Fig.23, that second from end pole of eight-pole wiggler according to (4) generates $\sim 1.34$ times bigger cubic nonlinearly integrated over pole, than the regular pole, as it has length $15 \mathrm{~cm}$ in contrast to $20 \mathrm{~cm}$ of regular one and about the same field. The end pole in seven-pole wiggler generates nonlinearity $\sim\left(\frac{20}{15}\right) \cdot\left(\frac{1.4}{2}\right)^{2} \cong 0.65$ of the main one, while the end pole of eight-pole wiggler generates nonlinearity what is $\left(\frac{20}{10}\right) \cdot\left(\frac{1}{2}\right)^{2} \cong 0.5$ of main pole. So summing up all poles effect for seven-pole wiggler we have $0.65+0.65+5=6.3$ units, meanwhile eight pole wiggler has $0.5+0.5+1.34+1.34+4=7.68$ units. So the eight-pole wiggler generates $7.68 / 6.3 \cong 1.22$ times more strong integrated cubic nonlinearity than a seven-pole one. So, roughly, five eight-pole wigglers give the same nonlinearity as six seven-pole ones. Full fifteen eight-pole wigglers will generate the same nonlinearity as eighteen one seven-pole ones. This is significant number. For reduction of nonlinear effect lowering vertical envelope function in region of wiggler can be used.

This nonlinearity might be important in case of intensive IBS scattering exciting big amplitudes of vertical oscillations as this keeps potential danger of drastic reduction of beam lifetime.

All eight-pole wigglers indicated mentionable dependence of second integral on direction of integration (with trims-off) $-35 \mathrm{~T} \cdot \mathrm{cm}^{2}$ over $2 \mathrm{~T} \cdot \mathrm{cm}^{2}$; this indicates that longitudinal asymmetry presents in the wiggler. Trims make this value evenly distributed only. This was not found for seven pole ones.

Seven poles wiggler was the first and technology of fabrication and assembling was not established yet. For example, the upper and lower halves were unparallel within $\sim 1.5 \mathrm{~mm}$ at one end, what was not acceptable, however the wiggler was installed due to time limits and desire to test the whole chain. Stainless steel plates became magnetized during annealing up to $\mu \sim 1.3$ in some places.

Discussion what number is better was caring from the very beginning and considered in [2]. Although even-pole idea was significantly transformed from its initial proposal: the wide poles accepted, shimming for flattening field roll off, tapering still it is not convincing. Final design of 
eight-pole wiggler has $22 \%$ more nonlinearity. Full tracking comparison will be represented in a separate publication.

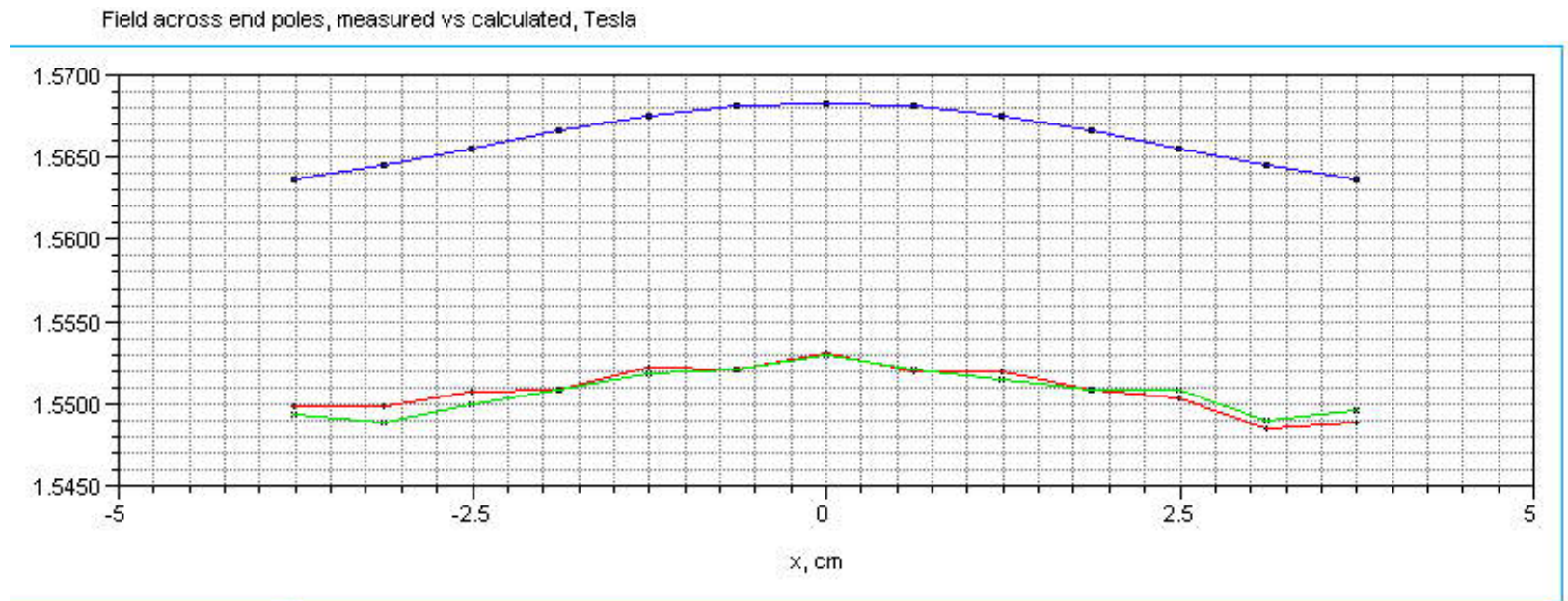

+ - End, mes, stack
$\times$ - End, mes, out
a -End, calc

FIGURE 24: Calculated and measured fields as functions of transverse coordinate. Wiggler \#1, end poles.

The difference between calculated and measured values can be estimated as 50G in absolute value. One can see from Fig.24, that end poles in wiggler \#1 are slightly twisted with respect to the medial plane. Geometrically, the StSteel plates shown if Fig. 20, were twisted by welding to about $1.5 \mathrm{~mm}$ gap at one side. In next wigglers welding performed with more rigid constrains and this plates are parallel. So that was a replica of the very first sample experience.

Measurements conclude with a series of files with measured data along lines defined by position of Hall probe in a cartridge, see Fig. 21. After that it is easy to capture correlations between data and compare with calculations.
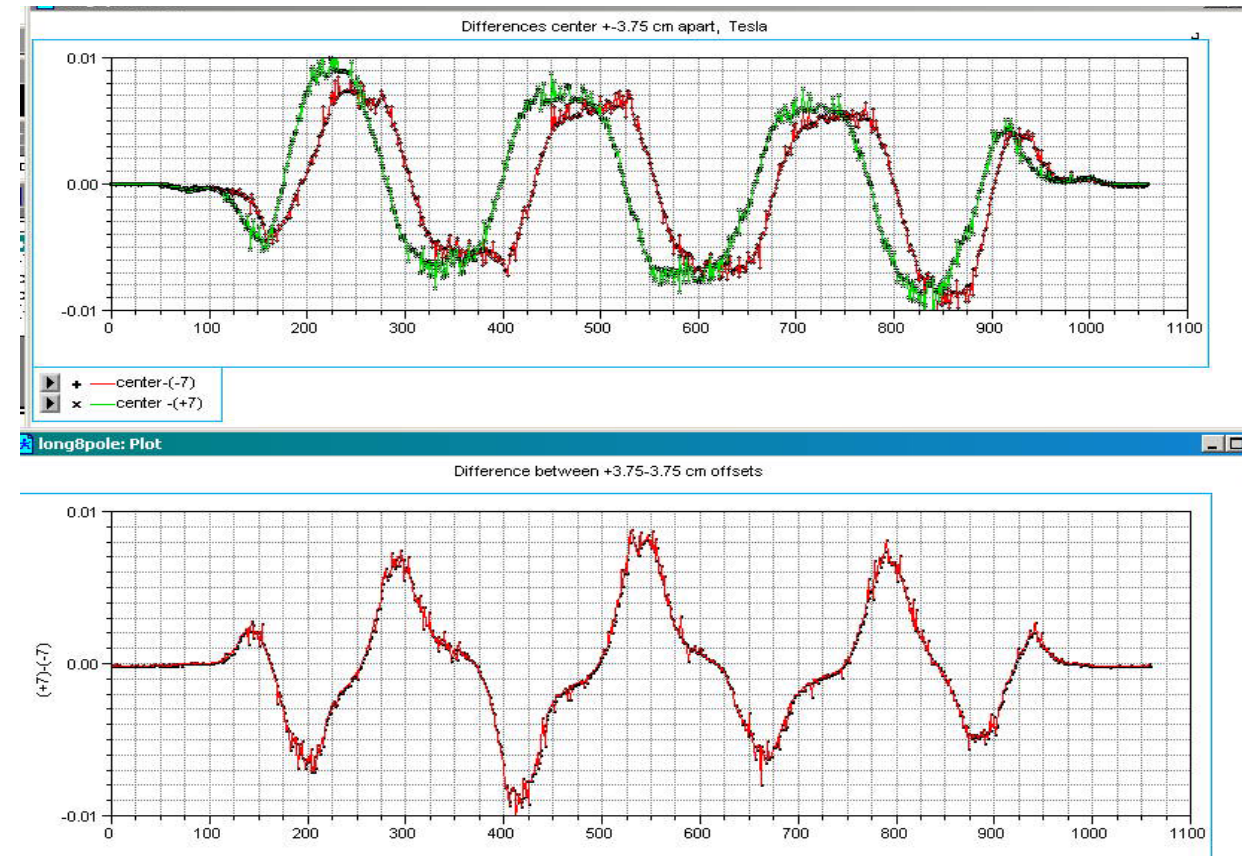

FIGURE 25: Longitudinal profile for $\pm 3.75 \mathrm{~cm}$ off-center lines in comparison. Eight pole wiggler $\# 5$. 
The curve, represented in Fig.25 is rather typical for all wigglers. It indicates that the cartridge is running slightly unparalleled to the wiggler longitudinal axes. Namely this is responsible for $\sim 80$ $\mathrm{G}$ difference between $+3.75 \mathrm{~cm}$ and $-3.75 \mathrm{~cm}$ Hall probe positions at the longitudinal slops of the field. This means that vacuum chamber is slightly misaligned with respect to the wiggler center.

\section{CONCLUSIONS}

Optimization of coil dimensions is the mostly difficult task. Each wiggler requires special efforts. Calculations with 3D code require much time and skills. MERMAID demonstrated excellent possibilities for such kind of tasks.

Tapering in a wiggler allows having zero displacement of trajectory. It might be vital for wigglers with even number of poles, as trajectory sweeps only half of transverse coordinate swept without tapering. This king of procedure can be recommended for TESLA damping ring, as one can see from publications, that they use even number of poles without proper tapering.

We represented here for the first time the possible tapering laws for zero displacement of orbit in a wiggler. This tapering law can be very useful for design of undulators and wigglers with smooth spectral characteristics.

Flatness of the poles need to be kept as plane as possible. This can be done either with profiling iron, either with active pole face winding. Practically all designs of wigglers with permanent magnets published so far suffering from underestimation of importance of flattering. In any case the poles need to be kept as wide as possible, limiting only by reasons of space and/or weight.

Measurements with long coils can be considered as indicative only, as they are not related directly to dynamic properties of the wiggler. Mapping with Hall probe can be considered acceptable only within moderate accuracy $\sim 10^{-3}$. With special probes having microchip with calibration, this can be lowered in half. Such probes are available on the market now.

For any wiggler operating at fixed field, angular kick in any given aperture region can be reduced to any desirable level, however.

Implementation of trim coils integrated with end poles of wiggler is extremely useful for fine tuning.

Nonlinearities in a wiggler strictly correlated with period, reversibly proportional to its square and proportionally to the square of magnetic field.

Confusion of past years initiated by measurements carried with long coils yield a narrow pole design, supported even more by choosing even number of poles. That is why real dynamic problems of the wiggler were recognized only recently.

In CESR wigglers the width of poles, $24 \mathrm{~cm}$ were made maximal possible and was defined by the diameter of Dewar, available at that moment. Request for operation within 1.7-2.1 Tesla made the wiggler pole flattening rather difficult task.

Pole face windings can be recommended for widening dynamic diapason.

Steel properties for room temperature deliver rather good approximation for such ones at liquid Helium temperature. 


\section{REFERENCES}

[1] CLEO-c AND CESR-c: A new Frontier of Weak and Strong Interactions, CLNS 01/1742, Cornell 2001.

G.Codner, G.Dugan, R.Ehrlich, Z.Greenwald, Y.He, S.Henderson, Y.Li, V.Medjidzade, A. Mikhailichenko, N.Mistry, E.Nordberg, J.Rogers, D.Rubin, D.Sagan, E. Smith, A. Temnykh, M.Tigner, Parameters for Low Energy operation of CESR, PAC2001, Chicago, IL June 18-22, 2001, Proceedings pp.374-376.

[2] A. Mikhailichenko, Wiggler for CESR operation at $2 \mathrm{GeV}$, Internal report, Cornell, http://cesrelog.lns.cornell.edu/documents/charm/param/am010122.pdf, Cornell U., 2000.

[3] A. Mikhailichenko, Optimized Wiggler Magnet for CESR, PAC2001, Chicago, IL, June 1822, 2001, Proc., pp. 3648-3650.

A. Mikhailichenko, The Wiggler for a Damping Ring, LC02, Feb. 4-8, SLAC, Stanford, CA, 2002.

[4] L. Smith, Effects of Wigglers and Undulators on Beam Dynamics, LB1-21391 (1986) and presented at the XIII Int.Conf. on High Energy ACC., Novosibirsk, USSR (1986).

[5] T. Raubenheimer, W.E. Gabella, P.L. Morton, Martin J. Lee, L.Z. Rivkin, R.D. Ruth, $A$ damping ring design for future linear colliders. (SLAC). SLAC-PUB-4912, Mar 1989. 3pp.

[6] G.Dudnikova, V.Vshivkov, K.Vshivkov, $U M K A-V G$, Institute of Computation Technologies, Lab. of Plasma Physics, Siberian Branch of RAN.

[7] A. Mikhailichenko, T. Moore, Lessons from 3-pole wiggler test, CBN 01-18, Cornell, LEPP, 2001.

[8] A. A. Mikhailichenko, V.V. Parkhomchuk, Damping Ring for a Linear Collider, BINP Preprint 91-79, Novosibirsk, 1991. 